of the 38 deaths due to pneumonia during the six weeks of the initial epidemic of the A2/Asian strain in 1957 , in only two was the infection purely staphylococcal, and an additional patient who died had mixed lesions which appeared to be both pneumococcal and staphylococcal. Apart from the four influenzal deaths, the remainder were pneumococcal (Forbes, 1958). This pattern has continued since.

The report, summarized in the same article, of $L$. $S$. Young describing a simultaneous outbreak of infection due to group $B$ meningococci and influenza amongst 55 elderly women in a mental institution in Mississippi is of interest in this context. A variety of interpretations is possible, but by analogy it seems likely that influenza potentiated invasion by the meningococei in a circum. stance of a high carriage rate.

Influenza thus appears to be an opportunistic killer to some extent, the ensuing mortality of an epidemic depending on the bacterial prevalence at the time and the availability of antibiotics.

\section{REFERENCES}

Forbes, J. A. (1958), "Severe Effects of Influenza Virus Infection", MED. J. AUBT., 2: 75.

LEAding ARTicle (1972), "Virus and Bacteria in Influenza", Brit. med. J., $2: 745$.

Newton-John, H. F., Yuna, A. P., Bennett, N. MCK., and Forbes, J. A. (1971), "Influenza Virus Pneumonitis: A Report of Ten Cases", MED. J. Aust., 2: 1160.

\title{
CONTROL OF INFLUENZA
}

\author{
Fred M. DavenPort, M.D., sc.med.d." \\ Department of Epidemiology, School of Public Health, University of Michigan, \\ Ann Arbor
}

Med. J. Aust. Spccial Suppl., 1973, 1: 33-38.

Control of influenza is yet to be accomplished. To do so requires the conquest of epidemics that recur with unpredictable severity and timing-events suffered since at least A.D. 1173 (Hirsch, 1883). To illustrate our plight, Figure 1 records the impact of influenza upon the population of the United States during the 36-year period when the causal viruses could be identified (Center for Disease Control, 1972).

Surveillance of influenza may be maintained by tabulating monthly pneumonia-influenza death rates, or by calculating excess mortality during epidemic periods. Excess mortality data register the excess of an expectancy calculated by fitting current reports to a theoretical curve which takes into account seasonal variations and long. term trends. This calculation for any particular epidemic provides but a minimal estimate of the havoc of that episode, since the contribution of the expected endemic level of deaths and the upper confldence limit thereof are automatically subtracted from the total. Nevertheless, the excess mortality index has been found practical and useful (Langmuir and Housworth, 1969).

\section{EPIDEMIC FREQUENCY}

If one looks for it, influenza is found annually in the United States, and quite properly, pneumonia-influenza deaths are reported each year (Davenport, 1958). The peaks and areas under the curves of the monthly

- Professor and Chairman, Department of Epidemiology School of Public Health, and Professor, Department of Internal Medicine, Medical School.

Address for reprints: Dr Fred M. Davenport, Chairman, Department of Epidemiology, School of Public Health, Univer sity of Michigan, 109 Observatory Street, Ann Arbor, Michigan, 48104 , U.S.A. pneumonia-influenza death rates appear to have declined somewhat gradually during the 1930's and early 1940's, but they have remained relatively constant during the past two decades. The decline is attributed to improved standards of living and to development of antibacterial therapeutics. The plateau indicates that widespread application of speciflc counter-measures will be required if further advances are to be made.

The majority of epidemics encountered since 1933 have been influenza $A$. The current epidemic of 1973 is not shown in Figure 1 , since the data are incomplete. In this 39-year period of record, 20 clear-cut outbreaks of influenza $A$ have occurred. The viruses involved have been designated A, A1, A2 and Hong Kong strains. These designations recognize the abrupt antigenic shifting which accompanies the emergence of a new subtype of influenza $A$ virus-an event which happens about every ten years. Its importance lies in the fact that vaccine efficacy is generally jeopardized by each major shift.

Eight epidemics of influenza $B$ have occurred during the same time period. Antigenic drift amongst strains of influenza $B$ takes place, but the degree of change is relatively minimal, and abrupt shifts permitting separation of strains into subtypes have not been encountered.

The high frequency of recurrence of epidemics is one of the most outstanding characteristics of influenza. On the average, $51.3 \%$ of the respiratory disease seasons represented in Figure 1 were marked by epidemics of influenza $A$ and $20.5 \%$ by epidemics of influenza $B$, and one or the other recurred in $71.8 \%$ of these respiratory disease seasons. To consider the data in another way, the odds are almost three to one that an epidemic of influenza will occur yearly. It is clearly futile to hide our heads in the sand and hope that influenza will go away; it will not. 
The severity of epidemics of influenza $A$ and $B$ has fluctuated widely since the early 1930 's, and without the development of clear trends we remain uncertain what to expect next. Either total excess mortality or excess mortality from pneumonia-influenza may be used to gauge the relative impact of different epidemics. The use of total excess mortality avoids the bias of change in fashions of reporting over time. The epidemics of influenza $A$ in 1936-1937 and 1943-1944 and of Asian influenza in 1957-1958 were outstanding catastrophes, as was that of influenza $B$ in 1935-1936. The remainder of the epidemics recorded, however, were far from trivial events. For example, while the first Asian epidemic of 1957 cost 69,800 excess deaths from all causes, the remaining epidemics through 1972 cost an additional 227,500 excess deaths for a total of 297,300 deaths. Truly, that year, as they arrived at training centres and were formed into companies. The remainder of each company received formalinized saline. Vaccine and placebo were allocated at random by use of the terminal digit of each recruit's serial number. Persons reporting to sick call with respiratory symptoms and fever were admitted to hospital, at which time throat swabs and admission blood samples were obtained. Convalescent samples were drawn two weeks later. The diagnosis of influenza was made by virus isolation, or more commonly by serological findings. The number of persons participating in each trial was large, generally in the thousands.

Table 1 summarizes the findings of the 14 trials carried out over the period indicated. The prevailing virus is designated by conventional nomenclature. Protection is expressed as the percentage reduction in influenza tound

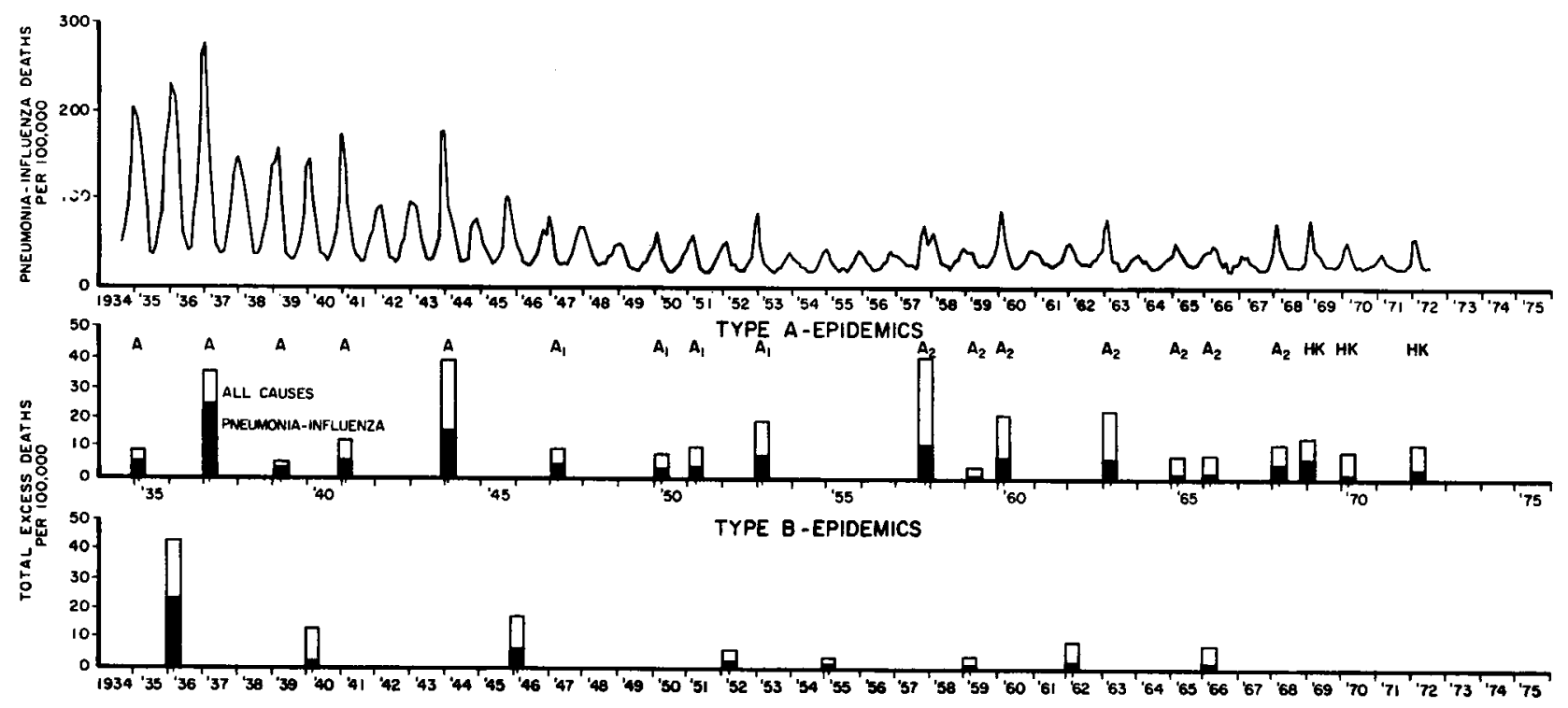

FIGURs 1: Pneumonia-influenza death rates by month, and excess mortality during epidemic periods, United States, 19341972. (From Epidemiology Program, Center for Disease Control.)

influenza is a recurrent killer of no small proportions. Yet death accounts for but a fraction of the total costs, and these when added up, stagger the imagination. For example, the relatively modest Hong Kong epidemic of 1968-1969 levied a total cost upon our population of $\$ 3,880,030,000$. At current prices, the cost of the 1957-1958 Asian epidemic would be $\$ 9,506,073,500$. Fortunately there are counter-measures.

\section{INFLUENZA VIRUS VACCINE FIELD TRIALS RESULTS}

The results of studies carried out over the past thirty years clearly demonstrate that properly constituted influenza virus vaccines confer a high degree of protection. In support of this statement, many sources of data could be used. The most extensive set are the results of field trials carried out by the Commission on Influenza of the Armed Forces Epidemiological Board in collaboration with the United States Army. The late Dr Thomas Francis junior designed and directed many of these carefully controlled studies, and kept a watchful eye upon them until his death. The basic plan was to vaccinate a fixed proportion of recruits with the vaccine under test among vaccinated subjects compared with the rate observed among controls. The results of the first two trials were gratifying-72\% protection against influenza $A$ and $92 \%$ against influenza $B$. The vaccine failure of 1947 is well known. Vaccination with A strains failed to induce protective antibodies, because the surface antigens of the prevailing A1 virus had undergone major antigenic shifting. When A1 vaccine was tested in 1950 , a high level of protection was again found. With one exception, excellent protection has been observed in all subsequent studies with other experimental vaccines. The exception was 1955 , when the epidemic $B$ strain of that year exhibited a significant antigenic drift away from the 1940 B Lee vaccine strain. Two failures in 27 years is not a bad record when so much had to be learned by trial and error. Considered in another way, the record for the last 14 years has been perfect.

Note that in the 12 successful trials, 7 of the vaccines yielded protection in the range of $80 \%$ to $90 \%$; an additional 5 vaccines gave protection at about the $70 \%$ level. Such levels of protection constitute a highly significant achievement, and they stand in sharp contrast to the malicious, yet oft-repeated statement: "Well after all, influenza virus vaccines are only about $40 \%$ to $60 \%$ effective." Those low estimates stem in part from the 
results of studies carried out with subjects unmatched for age, sex, housing, etc., and in which cases are counted by unreliable clinical criteria. Such studies yield diluted

Taber 1

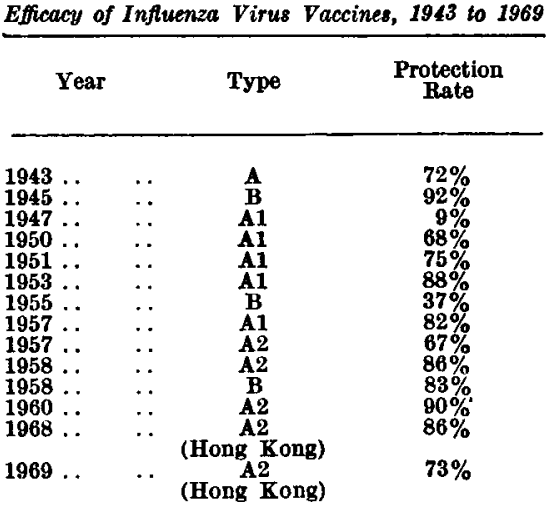

estimates of vaccine efficacy, especially when the relative incidence of influenza is low (Stuart-Harris, 1970).

An additional factor contributing to the high efficacy observed in these field trials is that the vaccines employed of the potency formerly used exclusively by the U.S. Army have now been adopted for protection of U.S. civilians.

CONTROL OF INFLUENZA BY ANNUAL VACCINATION

$A$ further demonstration of what can be accomplished by use of potent vaccines is shown in Figure 2 . The entire U.S. Army is vaccinated against infiuenza annually. The level of vaccination of civilians is unknown, but in most years the volume of vaccine produced is insufficient to vaccinate $10 \%$ of our population. In years of high promotional activity there is vaccine for at best $25 \%$.

In Figure 2, the index of involvement of the civilian population with influenza is the excess of influenza and pneumonia deaths plotted monthly so as to form a continuous line. The index of involvement of the Army is the annual morbidity rate of common respiratory disease and influenza per 1,000 average strength of Army personnel. These rates are plotted at the twenty-sixth week of each calendar year, with the exception that in 1957 the scale is truncated. Since the indices differ, they cannot be used to assess the relative impact of influenza upon the two populations. However, because they were uniformly applied to their respective populations, they can be used to measure differences in trends.

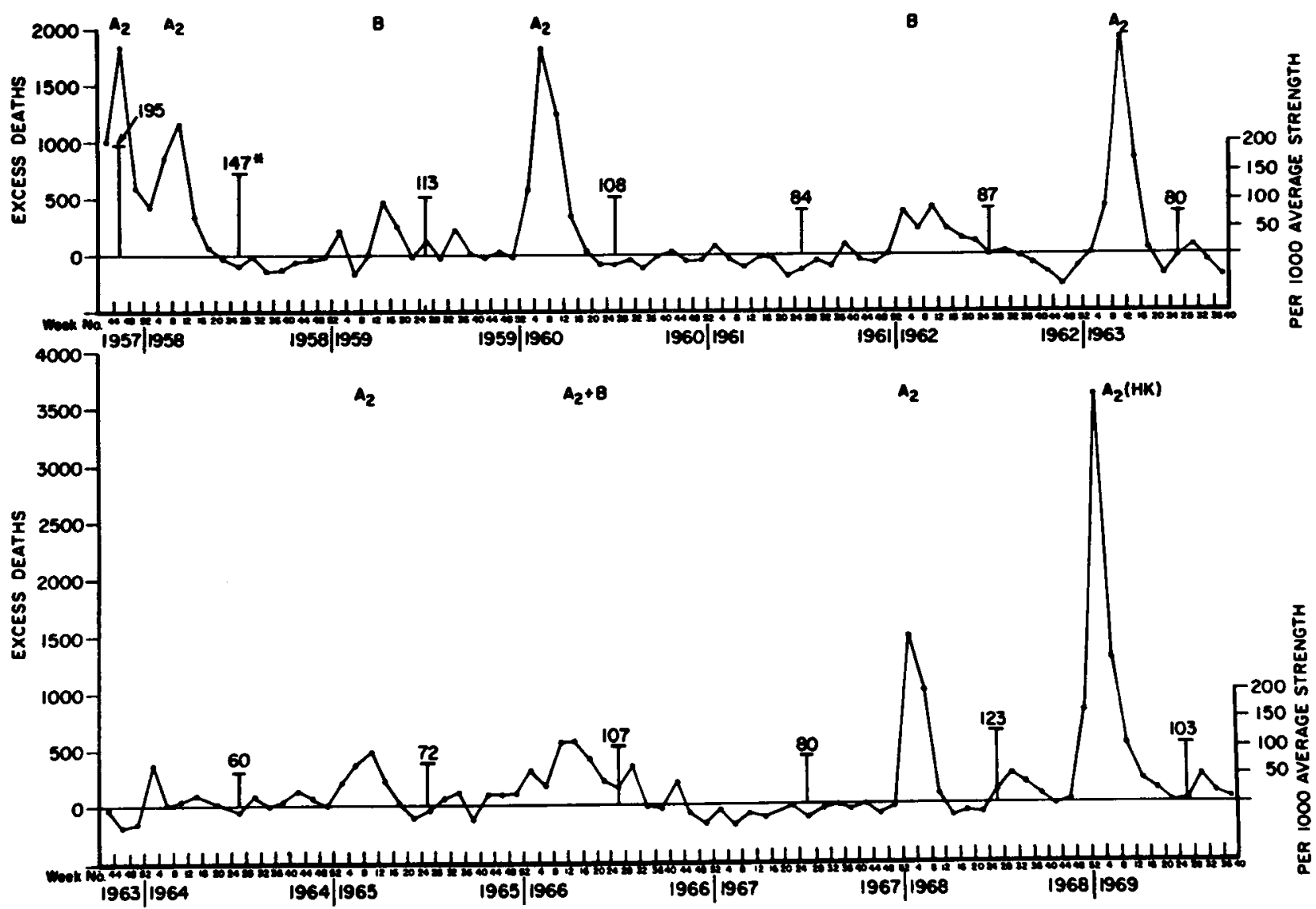

Figure 2: Excess pneumonia and influenza mortality, by four-week periods, from 122 United States cities during period of prevalence of $\mathrm{A} 2$ influenza. Asterisk indicates incidence of common respiratory disease and influenza per 1,000 average strength of Army personnel.

were special products obtained under contract from pharmaceutical laboratories. Invariably they contained a considerably larger dose of antigen than was present in vaccines released for civilian use. Fortunately, vaccines
Six sharp peaks mark the heavy involvement of the civilian population in $1957,1958,1960,1963,1968$ and 1969. A lesser degree of involvement is discernible in several of the other years. In contrast to the civilian 
experience, the incidence of common respiratory disease and influenza encountered by the army in epidemic years falls within the range observed in non-epidemic years. These are opposite trends. Clearly the annual use of influenza vaccines of adequate potency has brought influenza under control in our Armed Forces. The pro. nounced benefits of repeated vaccination observed in English boarding-schools add independent confirmation of the thesis that influenza can be controlled with vaccines now available once the decision has been made to use them properly (Turtle, 1968).

\section{REQUIREMENTS FOR CONTROL OF INFLUENZA}

If one concedes, then, that vaccination can control influenza in sectors of the population, the questions that remain with respect to total control are, "what is optimal strategy?" and "what are the other requirements?". A primary requirement is a change in attitude towards vaccination against influenza. Many persons either do not realize or else deny that influenza can be a devastating experience, and many physicians de-emphasize vaccination against influenza, either because of lack of knowledge concerning the benefits of vaccination or because of uncertainty about the availability of vaccine (Rosenstock, $1960)$.

Vaccine shortage is a recurrent problem in the United States, perpetuated by public pronouncements in which minimal levels of vaccine efficacy are consistently cited, the likelihood of an epidemic is depreciated or denied, and the caveat "Beware the consequences of antigenic shift ing" is sounded over and over again. In response to such tepid endorsements, manufacturers decide to make no more vaccine in that year than they are almost certain to sell. Programmes for education of the public and the medical profession concerning the benefits of vaccination are not undertaken, the reasoning being that it is unprofitable to encourage vaccination with a product that will not be available if the level of acceptance is raised. The outcome is that for the past 15 years we have been running in circles, without delivering a significant increase in benefits to the people we are supposed to serve. My concern is that, unless we break this vicious cycle and develop now a positive philosophy and programme for influenza control, the recurrence of a severe pandemic like that of 1889 or 1918 would find us virtually helpless once again.

An essential requirement for a positive programme is the assurance of an adequate supply of vaccine at all times. Stockpiling will be necessary to accomplish this objective. Despite steady improvements in speed of procurement of new epidemic strains and in vaccine production, the programme of capturing the latest antigenic variant in some unfortunate neighbour's backyard and of mounting crash programmes to obtain adequate supplies for oneself just does not work. Despite heroic efforts, the record is "too little, too late" (Murray, 1969). Size and composition of vaccine stockpiles are matters for internal decision conditioned by national policies and priorities. In making these decisions, the gamble must be taken that through antigenic shifting nature may or may not make one's stockpile obsolete once every ten years. I submit that nine-tenths of a loaf is better than none.

\section{STRATEGY FOR CONTROL OF INFLUENZA}

Once an adequate supply of vaccine is made available, the next question is how best to use it. At this point one must decide what is meant by control of influenza. In the U.S., the prime target has been defined as reduction of the shocking number of influenza-associated deaths. Persons at high risk of death during the annual periods of prevalence of influenza can be identifled. Primarily they are those of all ages who suffer from chronic debilitating conditions affecting the cardiovascular or bronchopulmonary systems, those with diabetes mellitus or other chronic metabolic disorders, and those without overt disease who are aged 55 years or older. Approximately $20 \%$ to $25 \%$ of our population fall into these categories, and about $\mathbf{4 0}$ million doses of vaccine would be required annually to afford protection for this sector of our population. Since the volume of vaccine produced annually in the United States is of the order of 20 million doses, and most of it is diverted for use in healthy people, it is clear that we are only paying lip service to the objective of reducing these deaths. One would not expect that these feeble efforts would exert a noticeable influence upon the number of influenza. associated deaths which occur in the U.S., and in point of fact they have not (Langmuir et alii, 1964). There is now available sufficient experience with mass vaccination programmes which could guide development of particulars for a successful campaign against influenza. What is missing is commitment to the task and assignment of an appropriate priority. Once these steps have been taken, we can honestly say that we have begun to try to control influenza.

However, the high-risk groups are only a part of the influenza problem. Their vaccination would clearly not interrupt the course of an epidemic, since they are not the most important spreaders. Schoolchildren are. This cohort generally experiences the highest attack rate, and has been incriminated as the source of transmission from schools to homes and thence to larger social agglegates (Jordan, 1960). Schoolchildren are readily available for vaccination once the public has been adequately prepared.

It has now been shown that vaccination of schoolchildren exclusively will afford a high degree of protection to the remainder of the community (Monto et alii, 1970). In the Fall of $1968,85 \%$ of schoolchildren enrolled in the first 12 grades at Tecumseh, Michigan, were vaccinated against Hong Kong influenza in four days. A control town, Adrian, was located 12 miles away. Administration of vaccine was virtually restricted to the 3,159 Tecumseh schoolchildren, because at that time Hong Kong vaccine was in short supply and available only for investigational use. Surveillance systems for disease reporting had long been operative in both towns. Hong Kong influenza struck Tecumseh and Adrian two weeks after vaccination had been completed. During the ten-week epidemic period, Adrian experienced three times as much acute respiratory illness as did Tecumseh. The findings shown in Figure 3 demonstrate that the benefit of vaccinating schoolchildren extended beyond the cohort vaccinated. It would be expected that the schoolchildren of Tecumseh would show a lower incidence of total acute respiratory disease, and they do; but note that, in addition, the rate of respiratory illness in each of the other age groups is higher in 
Adrian. The most marked difference is seen in the 20 to 29 years age group, reflecting the heavy exposure of young adults of Adrian to their unvaccinated school-age children.

Gratifying though this practical demonstration of the benefits of induced herd immunity is, it must be recognized that vaccination of schoolchildren will not completely protect persons in high-risk categories. At Tecumseh, viruses were isolated trom a number of persons aged over 55 years. Since death may be the consequence of exposure of unvaccinated high-risk subjects to influenza, vaccination of schoolchildren must be considered as an adjunct, not as a substitute, for vaccination of the chronically ill and the elderly.

Sixty million doses of vaccine would be needed to provide coverage of schoolchildren in our country. I am confident that mass vaccination of schoolchildren would provide a dramatic advance in the control of influenza, and that unless we take this step, efforts at total control are futile. It is possible that vaccination in schools would

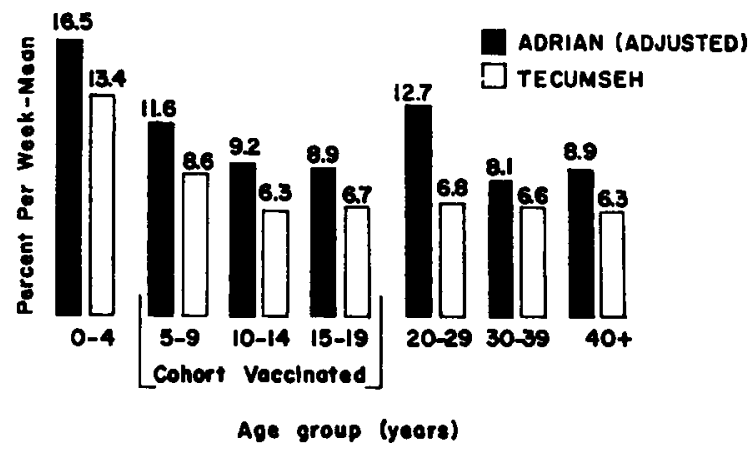

Figure 3: Survelllance population, Tecumseh and Adrian, Michigan: age-specific weekly mean rates of respiratory illness during the period of Hong Kong influenza.

not have to be carried out yearly on a routine basis, but could be postponed until times when epidemiological intelligence gave indication of the approach of a large outbreak. Experience over time could answer this question.

Once vaccination against influenza becomes more widely accepted, there will be enhanced interest on the part of industry and of those who prefer not to be ill to have their share in the benefits of a vaccine programme. Where the demand for vaccine will stop is difficult to say, but our capacity to produce vaccine would not be a limiting factor. All the vaccine need of the U.S. discussed today could be met by merely doubling or tripling the volume furnished during the six-month period in 1957 when vaccine production was at its peak.

\section{COST OF INFLUENZA EPIDEMICS}

Now, implementation of the strategy outlined would of course be costly, but what are the costs of an essen. tially do-nothing policy? Until recently there were no answers, but some are now becoming available. Kavet, in the U.S., has estimated certain of the costs of epidemic influenza utilizing the Dorothy Rice model of cost-benefit analysis (Kavet, 1972). A small portion of his findings is reproduced below:

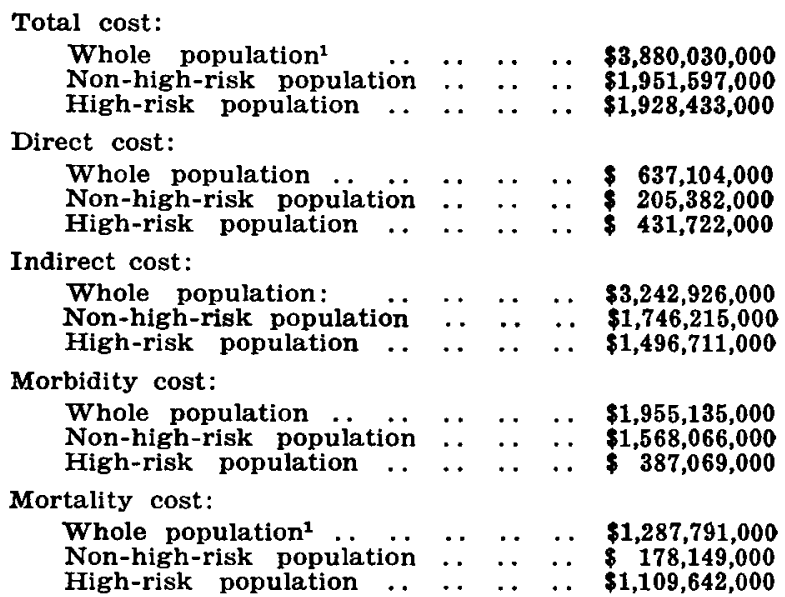

1 Mortality is expressed as the present value of future earnings lost discounted at $4 \%$.

Calculations were made with data relevant to the first Hong Kong epidemic of 1968-1969. As seen in Figure 1, that epidemic does not stand out as an especially' severe one.

The total cost of the epidemic was $\$ 3,880,030,000$. These are American billions, which modestly contain only 1,000 million each. The total cost for the non-high-risk population was slightly higher than that for the high-risk group. Clearly influenza is an important problem, not only for the elderly, but for those in the prime of life as well. Direct costs, which relate to delivery of medical care and to drugs, were considerable-\$637,104,000-and the majority of these dollars were spent in taking care of high-risk persons. Indirect costs represent an estimate of economic loss due principally to loss of earnings, production deficits and untimely deaths. These costs were not startlingly different between the groups. Together they account for the bulk of the cost of epidemic influenza -in this example, $\$ 3,242,926,000$. However, the characteristics of indirect costs in these two different segments of the population are quite different. The morbidity cost of the non-high-risk population was $\$ 1,568,066,000$, while the mortality cost was only $\$ 178,149,000$. That distribution of costs is strikingly different for the high-risk population $-\$ 1,109,642,000$ for mortality and only $\$ 387,069,000$ for morbidity.

The patterns of costs described are not unique to the U.S., and represent an enormous drain upon the economy of any country. Moreover, they are needless, since they are preventable. Control of influenza by appropriate vaccination programmes could be achieved by the investment of far less than the funds now being expended for medical services alone. These considerations, in addition to compassion for suffering and death, ought to motivate us as physicians and citizens to fight against apathy and ignorance, and to get on with the job of controlling influenza by intelligent use of the tools we now have at hand.

\section{ACKNOWLEDGEMENTS}

A portion of the investigations reported was conducted under the auspices of the Commission of Influenza, Armed U.S. Army Medical Research and Development Command, Department of the Army. 


\section{REFERENCES}

CENTER FOR DIBIAAE CONTROL (1972), Infuenza-Respiratory Disease Surveillance Report, No. 88.

Daven Port, F. M. (1958), "Symposium - Viruses: Current Advances with Clinical Applications; Recent Advances in Prevention of Influenza by Vaccination", Mod. Med., 26:115. Prevention of Influenza by Vaccination", Mod. Med., $26: 115$.
Hirscr, A. (1883), Handbook of Geographical and Historical Pathology, translated from the second German edition, New Sydenham Society, London, 1: 7 .

JordAN, W. S., JR (1960), "The Mechanism of Spread of Asian Influenza", Amer. Rev. resp. Dis., 83: 29.

Kavet, J. (1972), "Influenza and Public Policy", unpublished dissertation, Dr Sc., Harvard School of Public Health, Boston, Mass.

Lanamur, A. D., and Housworth, J. (1969), "A Critical Evaluation of Influenza Survellance", Bull. Wld Hhth Org., 41: 393.
Langaum, A. D., Hendraton, D. A, and Skrflina, R. E. (1964), "Epidemlological Basis for the Control of Influenza", A mer. J. publ. Hzth, $54: 563$.

Monto, A. S., DAVRNPORT, F. M., NAPIER, J. A., and Francis, J., JR (1970), "Modification of an Outbreak of Influenza in Tecumseh, Michigan by Vaccination of School Children". $J$. infect. Dis., $122: 16$.

MurRax, R. (1969), "Production and Testing in the U.S.A. of Influenza Virus Vaccine Made from Hong Kong Variant in 1968-69", Bull. Wld Hith Org., 41: 495.

Rosknstock, I. M. (1960), "Public Acceptance of Influenza Vaccination Programs" Amer. Rev. resp. Dis., 83: 171.

Stuart-Harris, C. H. (1970), "Control of Influenza: Lack of Knowledge Verus Lack of Application of Knowledge", Arch. environ. Hith, $21: 276$.

Turtur, P. DE BEC (1968), "Vaccines in the Management of Influenza Epidemics in Schools", Practitioner, 200: 254.

\title{
NEW APPROACHES TO THE CONTROL OF INFLUENZA
}

\author{
W. R. DOWDLE, PH.D.* \\ WHO International Influenza Center for the Americas, Center for Disease \\ Control, Atlanta, Georgia
}

Med. J. Aust. Special Suppl., 1973, 1: 38-41.

A Discussion of future developments in any field carries with it certain inherent elements of risk. The glowing promises of today's newly discovered techniques or newly proposed approaches to old problems often become only the dim promises of the future. I would like to avold these pitfalls as much as possible, and simply discuss with you what we need in the way of a better influenza vaccine, and some of the approaches now under consideration for producing that vaccine.

Wo might look upon this as an exercise in market analysis, in which we ask ourselves what it is that the public wants - or, to put it more bluntly, what it is that the public can be educated to want. Since I am also a member of the public, I have taken the liberty of listing some of those qualities of a vaccine which would seem to be essential if the product is to be successful. The ideal vaccine should have the following characteristics.

First, it must be desirable. The potential customerthat is, the public health authorities, the physicians, the general public - must be convinced that it serves a need.

Second, it must be acceptable. Any discomfort and Inconvenience - real or imagined-associated with taking the vaccine must be of considerably less magnitude than the discomfort - real or imagined - associated with the disease.

Third, it must be efficacious. It must provide a reasonable degree of protection for a reasonable period of time.

Fourth, it must be available. The customer must be able to obtain it when he needs it, and at a cost that he can afford.

Since the onset of the Asian influenza in 1957, a staggering 280,000 excess deaths have been associated with

- Director. Temporary address, Department of Microbiology, Australian National University, Canberra.

Address for reprinte : John Curtin School of Medical Research, A.N.U., Canberra, A.C.T. 2600 influenza in the United States alone; public health authori. ties clearly recognize the need for a vaccine. The total number of bed-days associated with influenza epidemics in the U.S. is more difficult to estimate, but it may approach 100 million in an epidemic season; the physician clearly recognizes the need for a vaccine. The average individual suffers from influenza of varying severity every four or five or even 10 years; he may not share the same enthusiasm for a vaccine.

In a study conducted in the U.S., just at the height of publicity of the impending 1957 epidemic, less than $50 \%$ of those surveyed believed that influenza would be more serious than the common cold. Less than $3 \%$ of the population believed that influenza would require any marked change in the individual's daily activity. The study concluded that a person would not take action to ward of a disease unless he believed, first, that he was susceptible to it, second, that its occurrence would be serious, and third, that effective and acceptable means for preventing or controlling it existed and were available to him.

To the patient with a chronic disease who has been convinced that influenza may be life-threatening, any inconvenience posed by the vaccine is of little consequence; but to the healthy and sceptical man in the street, the 30-year-old image of a partially effective vaccine with a high adverse reaction rate probably is not compatible with his concept of an acceptable means of preventing a mild disease which he may not get anyway. A new image for the raccine is essential.

One of the first aims in developing a new vaccine is to make it as reaction-free as possible. During the last few years there have, in fact, been a number of technical improvements in the production of the aqueous vaccines, which have led to a considerable reduction in toxicity. These may be summarized as follows: 